\title{
Communication Systems for High Speed Flying Devices with Repetition Codes
}

\author{
Dwi Juniarto $^{1,2}$, Khoirul Anwar ${ }^{1,2}$, Dharu Arseno ${ }^{2}$ \\ ${ }^{1}$ The Center for Advanced Wireless Technologies (AdWiTech), \\ ${ }^{2}$ School of Electrical Engineering, Telkom University, \\ \{dwijuniarto@student., anwarkhoirul@,darseno@\}telkomuniversity.ac.id
}

Manuscript received 16 January 2020; revised 20 February 2020; accepted 2 April 2020

\begin{abstract}
Communication systems for devices moving at high speed are suffering from error-floor due to the Doppler effect. This paper proposes a simple narrowband communication systems for high speed flying devices for critical applications such as missile and drone. To make the system simple, we consider Repetition codes and slight increase of the number of pilot symbols such that the system can predict accurately the fast-changing channel due to time-selective fading. The equalizer in this paper is designed according to the addition of the pilot symbols so that the system works at a maximum speed of $450 \mathrm{~km} / \mathrm{h}$ to make successful operation for missile and drone before they are taken down by the enemy. Computer simulations are used to evaluate the performance of the proposed communication systems. The operating frequency is industrial, scientific, and medical (ISM) band, where binary phase shift keying (BPSK) modulations are used with Repetition codes being the channel coding. The bit error rate (BER) performance is evaluated under additive white Gaussian noise (AWGN) and Rayleigh fading channels. The results confirm that excellent BER performances are obtained having error-floor less than $10^{-4}$ making many applications, including image transmission, are possible, which are great for high speed flying devices even with Repetition codes and simple zero forcing (ZF) equalizer. The results of this study are expected to help the development of future communication systems for missile, drone, and airplane applications.
\end{abstract}

Keywords: Doppler effect, pilot symbol, equalizer, BPSK, Repetition codes

DOI: $10.25124 /$ jmecs.v6i1.2459

\section{Introduction}

Wireless communication has advantages of providing services for devices with high mobility, such as flying devices. High mobility in wireless communication causes frequency shifts and phase changes, called the Doppler effect $[1,2]$, depending on the relative speed between transmitter and receiver [3, 4]. The frequency shift occuring due to the Doppler effect is one of problems to be considered, especially for high-speed communication systems for flying devices. Some high-speed flying device applications such as guided missiles and drones are expected to communicate well even they are moving at very high speed. Missile should be able to be controlled from the launcher without making mistake on hitting the target. Otherwise, it is dangerous if the mistake is just due to the communication errors. Similar to the case of missile, drone functioning for military applications should move in high speed and keep good communications with the controller on ground transmitting valuable information.

The frequency shift caused by the Doppler effect depends on the speed of movement of the transmitter or receiver and the carrier frequency. The higher the movement of speed and the frequency carrier, the greater frequency shift that occurs in the signal. However, if the carrier frequency is low, the frequency shift due to the Doppler effect is small [4].

The Doppler effect also causes channels to be timevarying or time-selective fading [5], i.e., channels that change very fast time-by-time. If the rapid change of this channel cannot be captured by the receiver, 


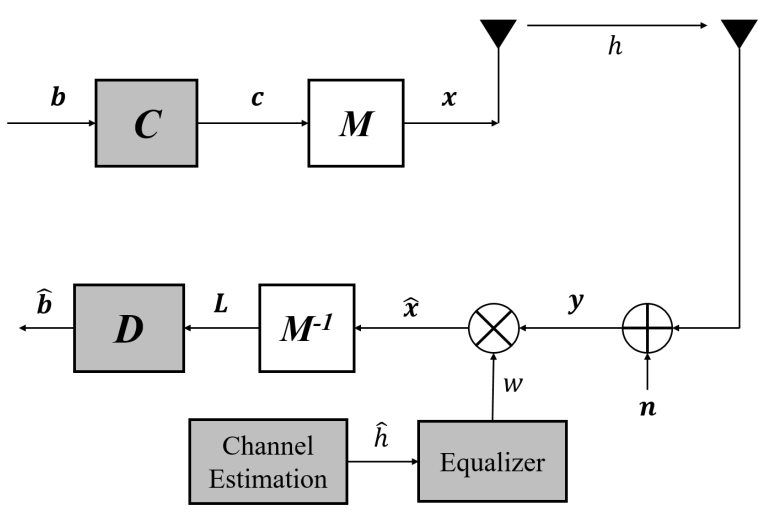

Fig. 1. The system model of communication systems for high speed flying devices, of which the proposed parts are indicated in darker blocks.

errors-floor appears because the signals are equalized using the wrong channel. Error-floor is a kind of error, which can not be reduced even though the noise is small. Because of this reason, channel equalization using the correct channel state information (CSI) is required to repair the signal damage caused by the high speed movement.

As shown in Fig. 1, an equalizer needs CSI $\hat{h}$ to perform channel equalization by providing weighting factor $w$. The purpose of the equalizer is to provide $w$ by finding the inverse of $\hat{h}$ to repair the signal damage caused by the differsive characteristic of the channels.

In this paper, we are targeting a maximum speed of $450 \mathrm{~km} / \mathrm{h}$ to make the missile or drone is difficult to be taken down by the enemy, where the channel $h$ is changing very fast within one block. Therefore, the channel should be estimated more frequently. However, too many pilot symbols are making the system loss in rate causing smaller transmission rate. To solve this problem, we propose only five pilot symbols are enough to estimate speed until $450 \mathrm{~km} / \mathrm{h}$. It should be noted here that the maximum $450 \mathrm{~km} / \mathrm{h}$ does not mean that the system is unoptimal for the speed below 450 $\mathrm{km} / \mathrm{h}$.

We found that not so many publications reporting the development of devices for military applications. Therefore, in this paper we perform comparison with the similar technique applied for public applications. Please note that the proposed technique is also applied for public applications.

Research in $[6,7,8]$ proposes equalization using turbo principle to overcome the intersymbol interference (ISI) in broadband communication without using guard interval or cyclic prefix (CP). The system can also be applied for communications having shorter $\mathrm{CP}$ length than the power delay profile (PDP) length.

Researches in $[9,10,11]$ have designed adaptive equalizer to reduce errors occuring at the receiver, which may require higher computational complexity.
However, since this paper considers the applications for flying devices, the level of complexity of a receiver [12] should be kept minimal. This paper designs communication systems for high speed mobile devices such as guided missiles, drones and airplanes.

Research [13] have evaluated the performances of Repetition codes and shown improvement in the Rayleigh fading environment. Research [14] have used Repetition codes with binary digital modulation, of which the results shown that Repetition codes can improve the performances on mobile channels.

Repetition codes are the simplest codes because the codes do not require memory or state diagram like in Convolutional codes or Turbo codes. The Repetition codes are expected to be useful for devices having small battery capacity. Even though Repetition codes are one of the simplest channel coding, Repetition codes can still provide performance improvement in fading channels, especially when soft decoding is applied.

The rest of this paper is organized as follows. Section 2. discusses the system model of the communications of flying devices. Section 3. performs zero forcing (ZF) and minimum mean square error (MMSE) equalization. In Section 4., we propose pilot-based channel estimation to track the changes of the channel state information (CSI) due to the fast moving of the flying devices. Performances are evaluated and analysed in Section 5.. Finally, the conclusions are provided in Section 6. with some concluding remarks.

\section{System Model}

Fig. 1 shows the system model of communication systems in this paper. Repetition codes are used as error-correction codes using soft decoding techniques. The coding rate used in Repetition codes is $R=\frac{1}{3}$. binary phase shift keying (BPSK) is used as the main modulation because it is simple and has the lowest error rate. Industrial, scientific, and medical (ISM) band used as a carrier frequency.

We consider narrowband communication systems between transmitter and receiver. The signal received by the receiver has been distorted by the channel and will be corrected by channel equalization. Channel estimation is needed to be able to do channel equalization. This paper uses pilot-assisted channel estimation to predict the channels that occur during the transmission. Zero forcing (ZF) and minimum mean squared error (MMSE) are used as algorithms for the equalizer on this system model. Signals that have been fixed by an equalizer are converted to log-likelihood ratio (LLR) values by demapper. The LLR value is used by the Repetition decoder to return to information bits because it is using soft decoding techniques.

We propose the use of Repetition codes as channel coding having coding rates $R=\frac{1}{2}$ and $R=\frac{1}{3}$. 


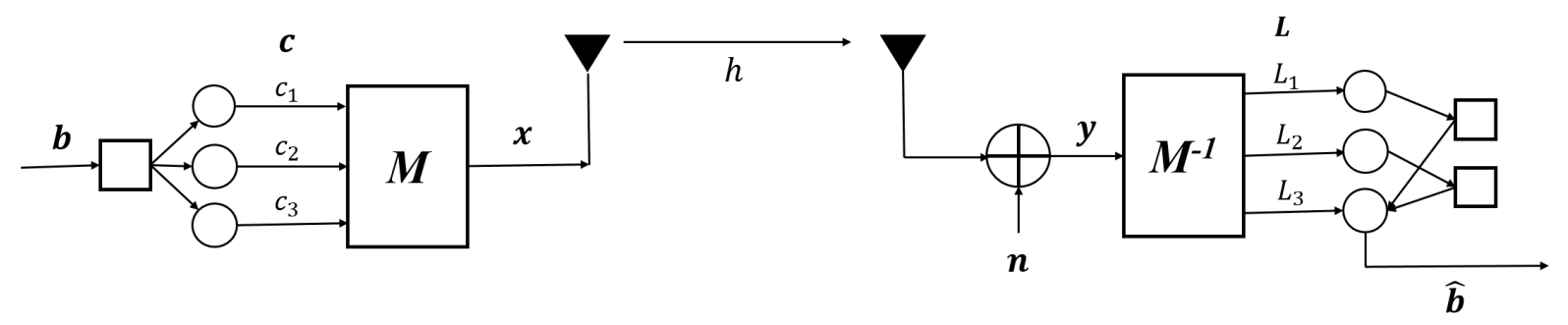

Fig. 2. Details of the proposed Repetition codes expressed in factor graph with rate $R=\frac{1}{3}$, where the "square" expresses check node and "circle" expresses variable nodes following $\mathbf{G}$ and $\mathbf{H}$ in (2) and (3) at the transmitter and receiver, respectively.

Fig. 2 illustrates the details of the Repetition codes with $R=\frac{1}{3}$. The proposed decoding technique used in this paper is the soft decoding. Therefore, the input for the Repetition decoder is a log-likelihood ratio (LLR) expressed with

$$
L=\frac{2}{\sigma_{n}^{2}} \cdot \operatorname{Re}(\mathbf{y})
$$

with $\sigma_{n}$ being the standard deviation of the additive white Gaussian noise (AWGN). The generator matrix for $R=\frac{1}{2}$ can be expressed as $\mathbf{G}=\left[\begin{array}{ll}1 & 1\end{array}\right]$. The parity check matrix for $R=\frac{1}{2}$ can be expressed as $\mathbf{H}=\left[\begin{array}{ll}1 & 1\end{array}\right]$.

Generator matrix for $R=\frac{1}{3}$ can be expressed with

$$
\mathbf{G}=\left[\begin{array}{lll}
1 & 1 & 1
\end{array}\right],
$$

of which the factor graph is shown at the transmitter side of Fig. 2 with three "circle" of variable nodes having one "square" check node. The actual number of variable nodes and check nodes is node three or one at the transmitter, however, the number is following the total block-length, which is the length information divided by $R$. The similar mechanism applies at the receiver side.

The parity check matrix for $R=\frac{1}{3}$ is expressed as

$$
\mathbf{H}=\left[\begin{array}{lll}
1 & 0 & 1 \\
0 & 1 & 1
\end{array}\right]
$$

of which the factor graph is shown at the receiver side of Fig. 2 having three variable nodes and two check nodes since the parity check matrix in (3) has two rows.

\section{Equalization}

Equalizer is a component at the receiver that is used to reduce or eliminate symbol interference (ISI) and recover damaged signals caused by channel distortion [15]. Channel estimation is needed in the equalizer. We use training or pilot data to estimate the channel. Equalizers can be classified in various ways, one of which is linear equalizer and non-linear equalizer. The linear equalizers are based on the channel delayed and do not have feedback. The output of this equalizer is linear combination of the inputs. The non-linear equalizers used to deal with large ISI and have a feedback. This paper evaluates the performance of linear equalizers namely ZF equalizer and MMSE equalizer.

\subsection{Zero Forcing Equalizer}

ZF equalizer is a linear equalizer. System model of linear equalizer is shown in Fig.1. Received signal at the receiver can be expressed in the frequency domain as

$$
\mathbf{y}=h \cdot \mathbf{x}+\mathbf{n},
$$

where $\mathbf{x}$ is transmitted symbol, $h$ is CSI obtained from pilot as in Figs. 4 and 5, and $\mathbf{n}$ is the AWGN noise. Fig. 6 shows how the CSI is more accurate when then number of pilot symbols is large.

Equalizer coefficient in $\mathrm{ZF}$ is an inverse function of channel estimation. ZF equalizer can be expressed as

$$
w=\hat{h}^{-1},
$$

where $\hat{h}$ is channel estimation [16]. The received signal $\mathbf{y}$ is recovered by the equalizer to get an estimation of the transmitted symbol that can be expressed as

$$
\hat{\mathbf{x}}=\mathbf{y} \cdot w .
$$

Equation (4) shows that the received signal y does not only contain the transmitted symbol, also contains noise, so that the estimated symbols in the receiver can be expressed as

$$
\begin{aligned}
\hat{\mathbf{x}} & =(h \cdot \mathbf{x}+\mathbf{n}) \cdot w \\
& =h \cdot \mathbf{x} \cdot \hat{h}^{-1}+\mathbf{n} \hat{h}^{-1}
\end{aligned}
$$

ZF equalizer has a disadvantage when the channel $h$ is of very low then the equalizer coeficient will be high. Fig. 3 [15] illustrates the amplification of noise that occurs when the channel is zero at the ZF and MMSE equalizers. Figs. 3(a), 3(b), and 3(c) show information, channel, and noise, respectively. Fig. 3(d) shows that the coefficient of $\mathrm{ZF}$ equalizer can be infinite value 


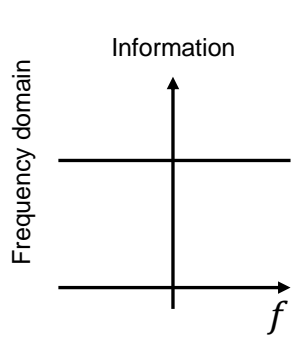

(a)

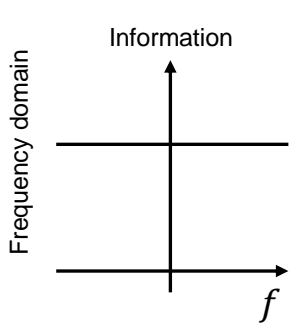

(f)

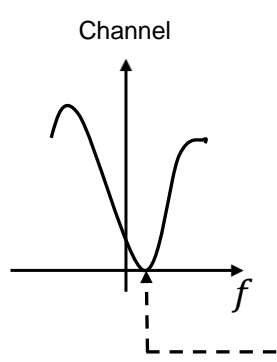

(b)

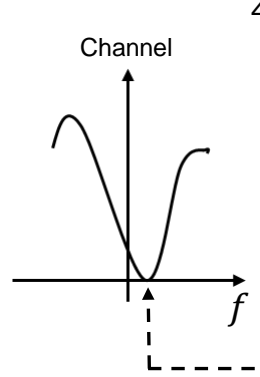

(g)

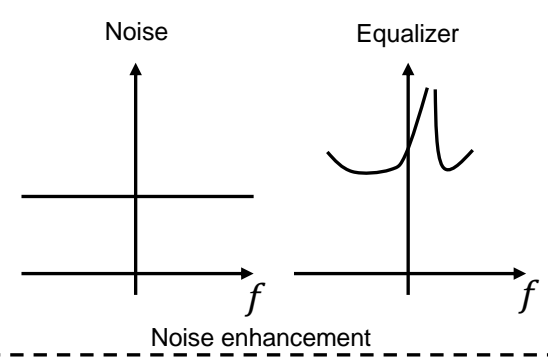

(d)

Zero Forcing Equalizer

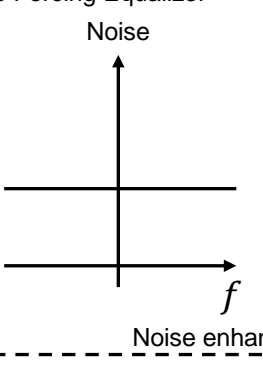

(h)

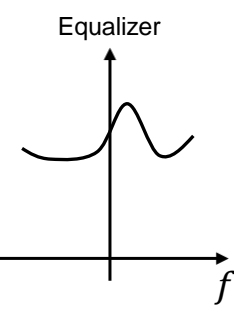

(i)

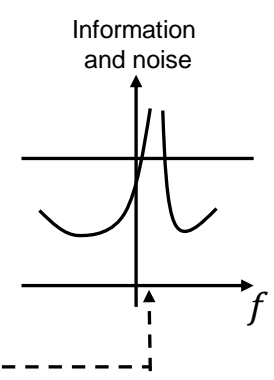

(e)

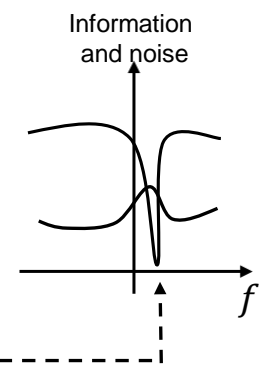

(j)

MMSE Equalizer

Fig. 3. The process of noise enhancement occuring in ZF and MMSE equalizers, where small noise results in infinity approaching weighting factor $w$.

when the channel is zero. Fig. 3(e) indicates that information has been returned to normal but when the channel is zero, the equalizer coefficient is infinite so the value of the noise also infinite. This shows that ZF not only reduces channel distortion but can also amplify noise significantly.

\subsection{Minimum Mean Squared Error Equalizer}

MMSE equalizer is a linear equalizer too. This paper explains how to get MMSE equalizer coefficient Fig. 1 show us that errors that occur can be expressed with

$$
\mathbf{e}=\mathbf{x}-\hat{\mathbf{x}} .
$$

Then the squared error value can be expressed with

$$
\mathbf{e}^{2}=\mathbf{x}^{2}-2 \mathbf{x} \mathbf{y}^{T} w+w^{T} \mathbf{y} \mathbf{y}^{T} w
$$

Then mean squared error value can be expressed with

$$
\begin{aligned}
E\left\{\mathbf{e}^{2}\right\}= & E\left\{\mathbf{x}^{2}-2 \mathbf{x} \mathbf{y}^{T} w+w^{T} \mathbf{y} \mathbf{y}^{T} w\right\} \\
= & E\left\{\mathbf{x}^{2}\right\}-2 E\left\{\mathbf{x} \mathbf{y}^{T}\right\} w \\
& +w^{T} E\left\{\mathbf{y} \mathbf{y}^{T}\right\} w .
\end{aligned}
$$

Then from the mean value, find the minimum value that can be expressed with

$$
\begin{aligned}
\frac{d E\left\{\mathbf{e}^{2}\right\}}{d w} & =-2 E\left\{\mathbf{x} \mathbf{y}^{T}\right\}+2 w E\left\{\mathbf{y} \mathbf{y}^{T}\right\}=0 \\
w & =E\left\{\mathbf{x y}^{T}\right\} E\left\{\mathbf{y y}^{T}\right\}^{-1} \\
& =R_{\mathbf{x y}} R_{\mathbf{y y}}^{-1}
\end{aligned}
$$

where $R_{\mathrm{xy}}$ is cross-corelation matrix between $\mathbf{x}$ and $\mathbf{y}$ and $R_{\mathbf{y y}}$ is auto-corelation matrix from $\mathbf{y}$. Transmitted signals $\mathbf{x}$ and noise $\mathbf{n}$ are independent then $E\{\mathbf{x n}\}=0$ and $E\{\mathbf{n x}\}=0$ so that $R_{\mathbf{x y}}$ and $R_{\mathbf{y y}}$ value can be expressed with

$$
\begin{aligned}
R_{\mathbf{x y}} & =E\left\{\mathbf{x} \mathbf{y}^{T}\right\} \\
& =E\left\{\mathbf{x}(h \mathbf{x}+\mathbf{n})^{T}\right\} \\
& =E\left\{\mathbf{x}\left(\mathbf{x}^{T} h^{T}+\mathbf{n}^{T}\right)\right\} \\
& =E\left\{\mathbf{x} \mathbf{x}^{T} h^{T}\right\}+E\left\{\mathbf{x n}^{T}\right\} \\
& =E\left\{\mathbf{x} \mathbf{x}^{T}\right\} h^{T} \\
& =\sigma_{x}^{2} h^{T},
\end{aligned}
$$

where $\sigma_{x}$ is variance of the received signal.

$$
\begin{aligned}
R_{\mathbf{y y}}= & E\left\{\mathbf{y} \mathbf{y}^{T}\right\} \\
= & E\left\{(h \mathbf{x}+\mathbf{n})\left(\mathbf{x}^{T} h^{T}+\mathbf{n}^{T}\right)\right\} \\
= & E\left\{(h \mathbf{x}+\mathbf{n})\left(\mathbf{x}^{T} h^{T}+\mathbf{n}^{T}\right)\right\} \\
= & \left.E\left\{h \mathbf{x} \mathbf{x}^{T} h^{T}+h \mathbf{x} \mathbf{n}^{T}+\mathbf{n} \mathbf{x}^{T} h^{T}+\mathbf{n} \mathbf{n}^{T}\right)\right\} \\
= & h E\left\{\mathbf{x} \mathbf{x}^{T}\right\} h^{T}+h E\left\{\mathbf{x n}^{T}\right\}+E\left\{\mathbf{n} \mathbf{x}^{T}\right\} h^{T} \\
& +E\left\{\mathbf{n n}^{T}\right\} \\
= & \sigma_{x}^{2} h h^{T}+\sigma_{n}^{2},
\end{aligned}
$$

where $\sigma_{n}$ is noise variance. From the values of $R_{\mathbf{x y}}$ and $R_{\mathrm{yy}}$ then MMSE equalizer coefficient can be ex- 


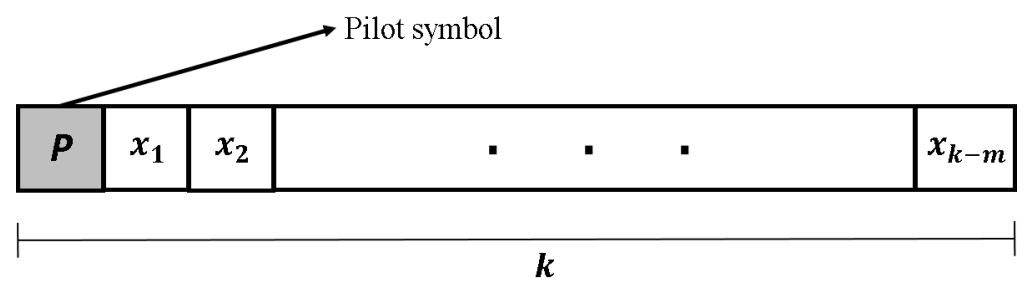

Fig. 4. One pilot symbol $P$ addition in the front of the frame resulting in the length of $k$ symbols.

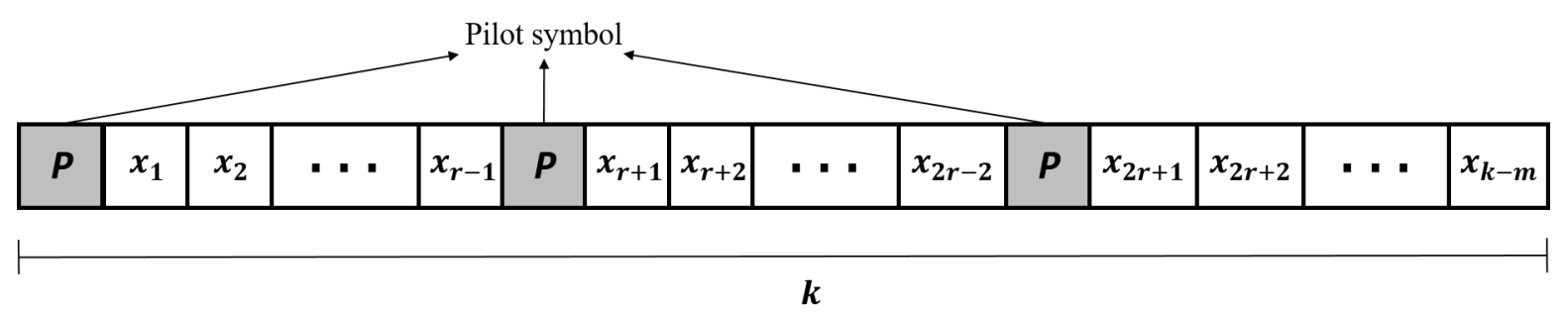

Fig. 5. Several pilot symbols $P$ addition to the frame resulting in total of length $k$ symbols.

pressed with

$$
\begin{aligned}
w & =\sigma_{x}^{2} h^{T}\left(\sigma_{x}^{2} h h^{T}+\sigma_{n}^{2}\right)^{-1} \\
& =\frac{h^{T}}{h h^{T}+\frac{\sigma_{n}^{2}}{\sigma_{x}^{2}}} .
\end{aligned}
$$

Figs. 3(f), 3(g), and 3(h) show information, channel, and noise, respectively. Fig. 3(i) shows the MMSE coefficient equalizer is not infinite when the channel is zero. Fig. 3(j) indicates that information has been returned but is not perfect and when the channel is zero, the equalizer is not infinite so there is no significant noise enhancement.

\section{Pilot-Assisted Channel Estimation}

In general, the communication systems uses only one pilot symbol for each frame shown in Fig. 4 with $k$ is the frame length and $m$ is the number of pilot symbols for each frame. This paper uses several pilot symbols so that the channel estimation in the system model is more accurate. Fig. 6 shows the different of channel estimation with one and five pilot simbols. Table 1 shows the parameters used in this paper. This paper determines the number of pilot symbols and the distance for each pilot symbol with the following calculation:

Table 1: The parameters used in this paper for high speed flying devices.

\begin{tabular}{l|l}
\hline Parameter & Value \\
\hline \hline Speed $(v)$ & $450 \mathrm{~km} / \mathrm{h}$ \\
\hline Carrier frequency $\left(f_{c}\right)$ & $915 \mathrm{MHz}$ \\
\hline frame length $(k)$ & 128 \\
\hline Bandwidth & $20 \mathrm{KHz}$ \\
\hline
\end{tabular}

- The maximum Doppler frequency in this paper is expressed by

$$
\begin{aligned}
f_{m} & =\frac{v \cdot f_{c}}{c} \\
& =\frac{120 \mathrm{~m} / \mathrm{s} \times 915 \cdot 10^{6} \mathrm{~Hz}}{3 \cdot 10^{8} \mathrm{~m} / \mathrm{s}} \\
& =366 \mathrm{~Hz} .
\end{aligned}
$$

- Based on [17], coherence time can be expressed with

$$
\begin{aligned}
T_{c} & =\frac{0.423}{f_{m}} \\
& =\frac{0.423}{366 \mathrm{~Hz}} \\
& =1.2 \mathrm{~ms} .
\end{aligned}
$$

- Table lindicates that the bandwidth is $20 \mathrm{KHz}$ so the symbol period can be expressed with

$$
\begin{aligned}
T_{s} & =\frac{1}{\text { Bandiwdth }} \\
& =\frac{1}{20 \cdot 10^{3} \mathrm{~Hz}} \\
& =50 \mu \mathrm{s} .
\end{aligned}
$$

- Then the number of pilot symbols needed by the system is expressed by

$$
\begin{aligned}
m & =\frac{T_{s} \times k}{T_{c}} \\
& =\frac{5 \cdot 10^{-5} s \times 128}{0.0012 \mathrm{~s}} \\
& =5.333 \\
& \approx 5 .
\end{aligned}
$$




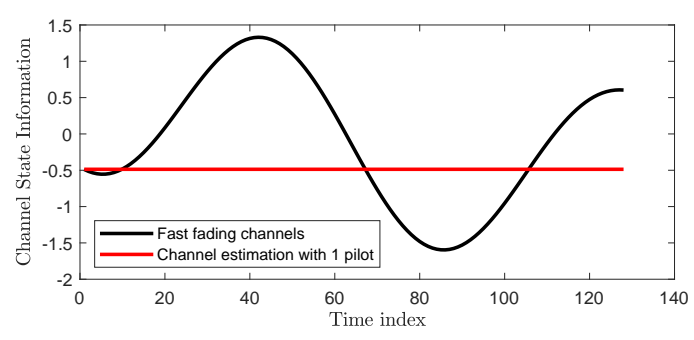

(a)

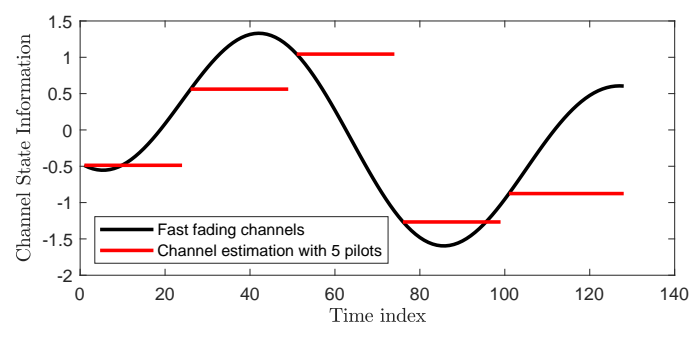

(b)

Fig. 6. Varying channel is estimated resulting channel estimate $\hat{h}$ (horizontal line) using: (a). one pilot symbol and (b). five pilot symbols.

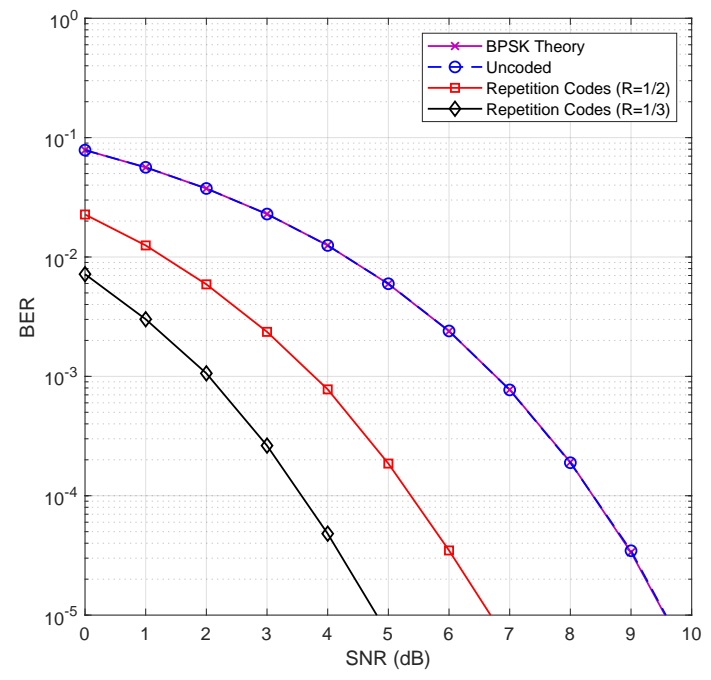

Fig. 7. BER performances of Repetition codes with different rates of $R=\frac{1}{2}$ and $R=\frac{1}{3}$.

- The distance for each pilot on the system is expressed by

$$
\begin{aligned}
r & =\frac{k}{m} \\
& =\frac{128}{5} \\
& =25.6 \\
& \approx 25 .
\end{aligned}
$$

\section{Performance Evaluation}

\subsection{BER Analysis of Repetition Codes}

Fig. 7 shows the BER curve for a system without channel coding and system with Repetition codes on the AWGN channel. The simulation using BPSK modulation and soft decoding technique in the Repetition decoder. Fig. 7 using BPSK theory as a basic reference. "Uncoded" curve is simulation result without using channel coding and the result obtained is in

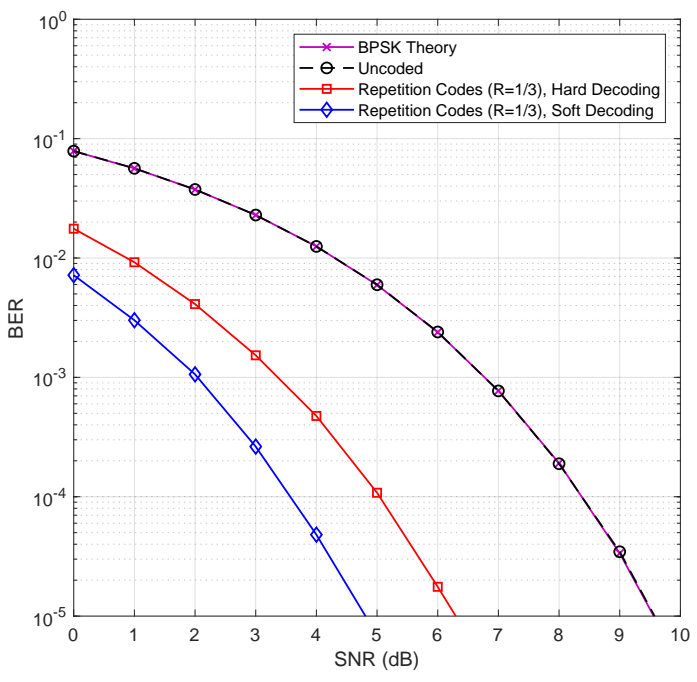

Fig. 8. BER performances of Repetition codes with different decoding techniques of hard and soft decodings.

accordance with BER theory. This proves that the simulation of the system model for uncoded is validated.

System without channel coding, BER $10^{-4}$ is achieved at SNR $\gamma=8.37 \mathrm{~dB}$. On system with Repetition codes with rate $R=\frac{1}{2}$, BER $10^{-4}$ is achieved at SNR $\gamma=5.37 \mathrm{~dB}$ and system with Repetition codes with rate $R=\frac{1}{3}$ is achieved at $\mathrm{SNR} \gamma=3.57 \mathrm{~dB}$. System with Repetition codes has a better performance $3 \mathrm{~dB}$ for rate $R=\frac{1}{2}$ and $4.8 \mathrm{~dB}$ for rate $R=\frac{1}{3}$ at BER $10^{-4}$ compared to system without using channel coding.

\subsection{BER Analysis of Decoding Technique}

Fig. 8 shows the BER curve for a system without hard decoding technique and system with soft decoding technique on the AWGN channel. The simulation using BPSK modulation and coding rate $R=\frac{1}{3}$. System with hard decoding technique, BER $10^{-4}$ is achieved at SNR $\gamma=5.04 \mathrm{~dB}$ and system with soft 


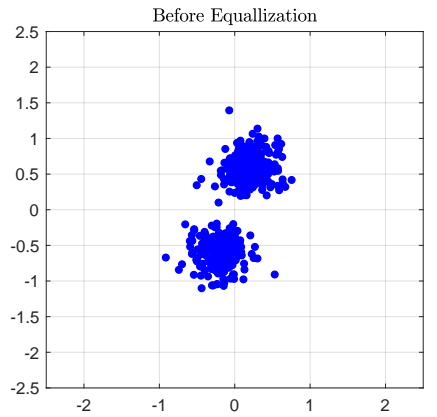

(a)

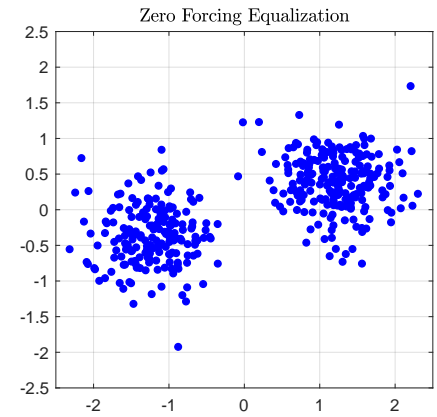

(b)

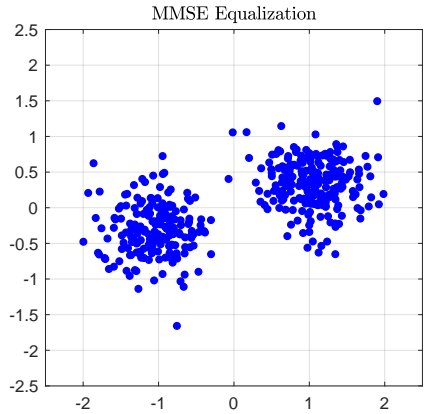

(c)

Fig. 9. Constellation diagram for: (a). Symbols before equalization, (b). Symbols after ZF equalization, and (c). Symbols after MMSE equalization.

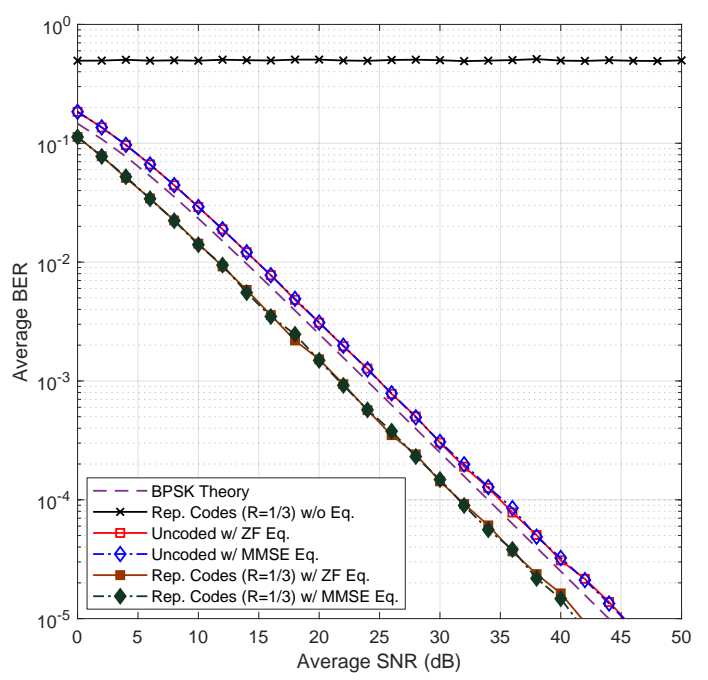

Fig. 10. BER performances of ZF and MMSE equalizers.

decoding technique is achieved at SNR $\gamma=3.57$ $\mathrm{dB}$. System with soft decoding technique has a better performance of $1.47 \mathrm{~dB}$ at BER $10^{-4}$ compared to system with hard decoding technique.

\subsection{Equalizer Analysis}

Fig. 9 shows that the symbol amplitude using ZF equalizer is more distorted compared to MMSE equalizer. This will be clearer when the channel coefficient that occurs is zero or very small because ZF not only improves the signal but also amplifies the noise significantly whereas MMSE overcomes this problem. However, in the case of they are correcting the error in phase, both equalizers have the same performances. Suppose that the ZF coefficient of equalizer is $\hat{h}=a+j b, j=\sqrt{-1}$, based on (5) the phase for
$\mathrm{ZF}$ equalizer can be expressed as

$$
\theta=-\tan ^{-1}\left(\frac{b}{a}\right) .
$$

As for the MMSE equalizer, the denominator value based on (14) is real value. So that the phase of the MMSE equalizer is only affected by the numerator. Suppose $\hat{h^{*}}=a-j b$, phase for MMSE equalizer can be expressed as

$$
\theta=\tan ^{-1}\left(-\frac{b}{a}\right)
$$

Fig. 10 using BPSK fading theory as a basic reference. BER for systems without equalizer has bad performance with error constant at BER 0,5. However, a system with an equalizer and Repetition codes can change errors better than a system with an equalizer and without channel coding of $4 \mathrm{~dB}$ for almost all SNR. Fig. 10 shows the BER curve with ZF equalizer and with MMSE equalizer has the same results. This shows that the amplitude of the symbol which is more distorted using ZF in BPSK modulation does not have much effect when deciding the symbol in the demapper so it does not affect the performance of the system model. It also shows that the phase is very influential when deciding symbols for BPSK modulation in demapper.

\subsection{BER Analysis of The Proposed Communica- tion Systems}

Fig. 11 shows the BER curve from the systems at speed $v=120 \mathrm{~km} / \mathrm{h}$. Simulations are carried out using BPSK modulation. Systems without Repetition codes and with one pilot symbol starts an error-floor at $5 \times 10^{-5}$ at SNR $\gamma=44 \mathrm{~dB}$. While systems with Repetition codes and one pilot symbol also start an error-floor at $5 \times 10^{-5}$ at SNR $\gamma=44 \mathrm{~dB}$. Systems without Repetition codes and with five pilot symbols starts an error-floor at $7.5 \times 10^{-6}$ at SNR $\gamma=48$ 


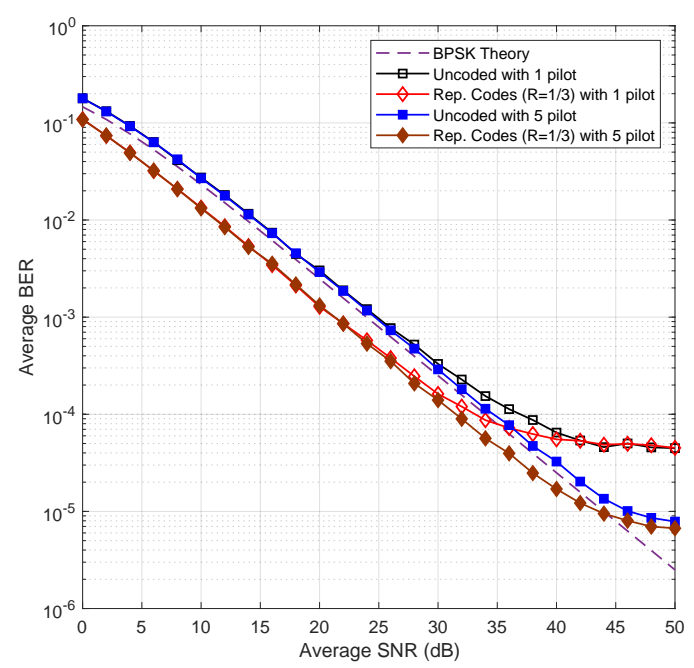

Fig. 11. BER performances of system with pilot symbols at $v=120 \mathrm{~km} / \mathrm{h}$.

dB. While systems with Repetition codes and five pilot symbols also start an error-floor at $7.5 \times 10^{-6}$ at SNR $\gamma=48 \mathrm{~dB}$. Systems with Repetition codes has a better performance of $4 \mathrm{~dB}$ for almost all SNR before an error-floor. This shows that the error-floor cannot be fixed by Repetition codes but can be corrected by modifying the equalizer.

Fig. 12 shows the BER curve from the systems at speed $v=450 \mathrm{~km} / \mathrm{h}$. Simulations are carried out using BPSK modulation. Systems without Repetition codes and with one pilot symbol starts an error-floor at $6 \times 10^{-4}$ at SNR $\gamma=36 \mathrm{~dB}$. While systems with Repetition codes and one pilot symbol also start an error-floor at $6 \times 10^{-4}$ at SNR $\gamma=36 \mathrm{~dB}$. Systems without Repetition codes and with five pilot symbols starts an error-floor at $5.7 \times 10^{-5}$ at SNR $\gamma=45 \mathrm{~dB}$. While systems with Repetition codes and five pilot symbols also start an error-floor at $5.7 \times 10^{-5}$ at SNR $\gamma=45 \mathrm{~dB}$. This results proves that the error-floor can still be fixed by the equalizer even though the speed increases.

Fig. 12 indicates that we can reduce the threshold of error-floor from $6 \times 10^{-4}$ to $5.7 \times 10^{-5}$ making more reliable applications are possible. Application are depending on the threshold of the communication between missile/drone and the controller. If the communication is only for text, then BER $10^{-3}$ is enough. However, in the proposed system we are targeting the communication including seeker to track the target requiring excellent communication between missile/drone and the controller for image transmission. Therefore, we are targeting the BER should be less than $10^{-4}$, which is achieved by the proposed techniques.

Fig. 13 shows the BER curve versus $f_{d} T_{s}$ for SNR

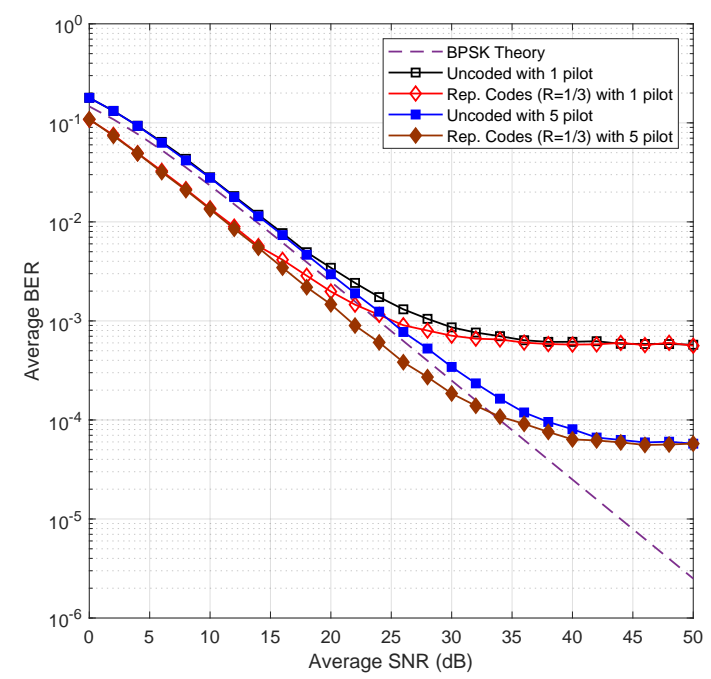

Fig. 12. BER performances of system with pilot symbols at $v=450 \mathrm{~km} / \mathrm{h}$.

$\gamma=25 \mathrm{~dB}$ and SNR $\gamma=50 \mathrm{~dB}$ where $f_{d} T_{s}$ is normalized Doppler spread over the single carrier symbol duration. System with Repetition codes and one pilot symbol at SNR $\gamma=25 \mathrm{~dB}$ and SNR $\gamma=50 \mathrm{~dB}$, BER $10^{-2}$ is achieved at $f_{d} T_{s}=6.1 \times 10^{-4}$ and system with Repetition codes and five pilot symbol is achieved at $f_{d} T_{s}=2 \times 10^{-3}$. This results proves that, if speed is increased, errors occur faster on systems with one pilot symbol compared to systems with five pilot symbols.

\section{Conclusion}

This paper has proposed simple a communication system for high speed flying devices with maximum speed of $450 \mathrm{~km} / \mathrm{h}$, where Repetition codes are used as channel decoding because of its simplicity. This paper has also proposed multiple pilot symbols to improve accuracy of channel estimation. To keep the system has low computational complexity beside (a) the use of Repetition codes, whe have also (b) proposed keeping five pilot symbols to track the change of channel state information accurately and (c) to use ZF equalizer since the performance of ZF for BPSK have similar performances to that of MMSE equalizer, of which ZF is suitable for high speed flying device. The results of this paper are expected to provide contributions to the development of reliable communications for high speed flying devices.

\section{Acknowledgment}

This work is in part the output of the ASEAN IVO (http://www.nict.go.jp/en/asean_ivo/index.html) project PATRIOT-41R-Net 2019-2021, which is financially supported by NICT (http://www.nict.go.jp 


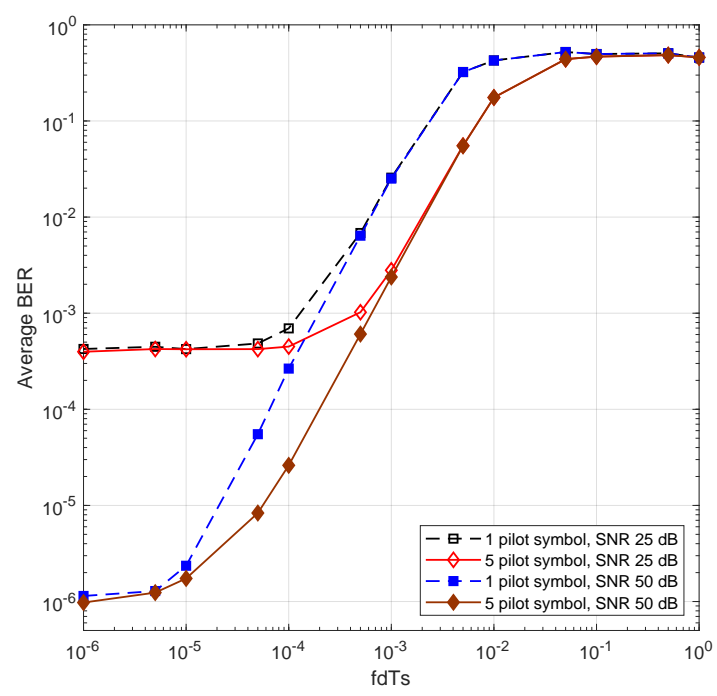

Fig. 13. BER performances of communication systems of one and five pilot symbol versus normalized Doppler spread $f_{d} T_{s}$.

/en/index.html).

\section{References}

[1] J. Schiller, Mobile Communications, 2nd ed. Great Britain, 2003.

[2] C. He, J. Huang, and Q. Zhang, "Research on bandwidth efficient underwater acoustic communications," in TENCON 2010 - 2010 IEEE Region 10 Conference, Nov 2010, pp. 742-745.

[3] Z. Zhao, "Wi-Fi in high-speed transport communications," in 2009 9th International Conference on Intelligent Transport Systems Telecommunications, (ITST), Oct 2009, pp. 430-434.

[4] H. Tavakoli, M. Ahmadian, Z. Zarei, and M. Zourabadi, "Doppler effect in high speed," in 2008 3rd International Conference on Information and Communication Technologies: From Theory to Applications, April 2008, pp. 1-5.

[5] T. J. Riedl and A. C. Singer, "Broadband Doppler compensation: Principles and new results," in 2011 Conference Record of the Forty Fifth Asilomar Conference on Signals, Systems and Computers (ASILOMAR), Nov 2011, pp. 944-946.

[6] H. Zhou, K. Anwar, and T. Matsumoto, "Chained turbo equalization for SC-FDMA systems without cyclic prefix," in 2010 IEEE Globecom Workshops, Dec 2010, pp. 1318-1322.

[7] K. Anwar, Z. Hui, and T. Matsumoto, "Chained turbo equalization for block transmission with- out guard interval," in 2010 IEEE 71st Vehicular Technology Conference, May 2010, pp. 1-5.

[8] A. Irawan, K. Anwar, and T. Matsumoto, "Chained turbo equalization for multiuser SIMOOFDM systems without cyclic prefix," in 2012 International ITG Workshop on Smart Antennas (WSA), March 2012, pp. 301-306.

[9] Y. Nan and X. Sun, "Design and implementation of adaptive equalizer in tetra digital trunked system," in 2012 Second International Conference on Instrumentation, Measurement, Computer, Communication and Control, Dec 2012, pp. 1674-1677.

[10] G. D'Aria, R. Piermarini, and V. Zingarelli, "Fast adaptive equalizers for narrow-band TDMA mobile radio," IEEE Transactions on Vehicular Technology, vol. 40, no. 2, pp. 392-404, May 1991.

[11] S. M. K. and, "Broadband adaptive noise equalizer," IEEE Signal Processing Letters, vol. 3, no. 8, pp. 234-235, Aug 1996.

[12] G. Ungerboeck, "Adaptive maximum-likelihood receiver for carrier-modulated data-transmission systems," IEEE Transactions on Communications, vol. 22, no. 5, pp. 624-636, May 1974.

[13] W. C. Y. Lee, "The advantages of using repetition code in mobile radio communications," in 36th IEEE Vehicular Technology Conference, vol. 36, May 1986, pp. 157-161.

[14] A. A. Ali and I. A. Al-Kadi, "On the use of repetition coding with binary digital modulations on mobile channels," IEEE Transactions on Vehicular Technology, vol. 38, no. 1, pp. 14-18, Feb 1989.

[15] A. F. Molisch, Wireless Communications, 1 st ed. IEEE, 2011.

[16] H. Kim, Wireless Communications Systems Design, 1st ed. Wiley, 2015.

[17] Y. S. Cho, J. Kim, W. Y. Yang, and C. G. Kang, MIMO-OFDM Wireless Communications with $M A T L A B, 1$ st ed. Wiley-IEEE Press, 2010.

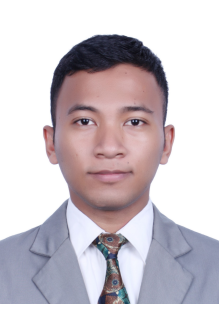

Dwi Juniarto is pursuing Bachelor degree program of School of Electrical Engineering, Telkom University. Currently, Dwi is research member of the Center for Advanced Wireless Technologies (AdWiTech), Telkom University 
researching wireless communications for high speed flying devices. His research interest is on channel coding, equalizer, channel estimation, and hardware implementation.

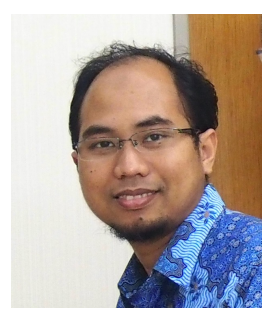

Khoirul Anwar (SM'16) received the bachelors degree (cum laude) from the Department of Electrical Engineering (Telecommunications), Bandung Institute of Technology, Bandung, Indonesia, in 2000, and the master's and Ph.D. degrees from the Graduate School of Information Science, Nara Institute of Science and Technology, Nara, Japan, in 2005 and 2008, respectively. He was with the University of Melbourne, Australia, in 2007, the University of Oulu, Finland, in 2010, and Cranfield University, U.K., in 2018, as a Visiting Researcher. In 2008, he was with the School of Information Science, Japan Advanced Institute of Science and Technology, as an Assistant Professor. Since 2016, he has been with the School of Electrical Engineering, Telkom University, Bandung, as an Associate Professor, and the Director of the Center for Advanced Wireless Technologies.

He has been the Chairman of WG Radio and Technologies of Indonesia 5G Forum and the Chairman of the Asia Pasific Telecommunity Wireless Group on Service and Applications, 2016-2019. Dr. Anwar is currently the Vice-chairman of the Asia Pasific Telecommunity Wireless Group, 2019-2021.

His research interests are network information theory, error correction coding, iterative decoding, coding for super-dense networks, and signal processing for wireless communications. His technique is adopted by the international telecommunication union (ITU), ITUR standard No. ITU-R S.2173 Multi-Carrier-Based Transmission Techniques, and ITU-R S.1878 Multicarrier Based Transmission Techniques for Satellite Systems. He is a Senior Member of the IEEE Information Theory Society and Communications Society.

He received the Best Student Paper Award at the IEEE Radio and Wireless Symposium 2006 (RWS06), CA, USA, the Best Paper Award of Indonesian Student Association (ISA 2007), Kyoto, Japan, in 2007, the Best Paper Presenter for the Advanced Technology at the International Conference on Sustainability for Human Security (SUSTAIN), Kyoto, in 2011, the Indonesian Diaspora Award for Innovation, Congress of Indonesian Diaspora, Los Angeles, USA, in 2012, the Achmad Bakrie Award 2014, Jakarta, in 2014, and the Anugerah of Internationally Recognized Contributions from the Governor of West Java, Indonesia, in 2016, the National Achievement Award from UKP-PIP Pan- casila, Jakarta, in 2017, OCBC award in August 2019. He serves as a reviewer for a number of main journals and conferences in the areas of wireless communications, coding theory, and signal processing.

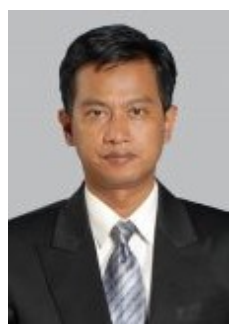

Dharu Arseno received bachelor degree and masters degree from the Departement of Electrical Engineer- ing, Bandung Institute of Technology (ITB) in 1995 and 2001, respectively. Dharu is currently a senior lecturer at School of Electrical Engineering, Telkom University since 2002. His research interest is on wireless communications and radar 